\title{
Polyethylenimine-mediated expression of transgenes in the acinar cells of rats salivary glands in vivo
}

\section{Monika Sramkova ${ }^{\dagger}$, Laura Parente $^{\dagger}$, Timothy Wigand, Myo-Pale' Aye, Akiko Shitara and Roberto Weigert*}

Intracellular Membrane Trafficking Unit, Oral and Pharyngeal Cancer Branch, National Institute of Dental and Craniofacial Research, National Institutes of Health, Bethesda, MD, USA

\section{Edited by:}

Ken William Dunn, Indiana

University School of Medicine, USA

Reviewed by:

Heike Folsch, Northwestern

University Feinberg School of

Medicine, USA

Robert Bacallao, Indiana University

School of Medicine, USA

\section{${ }^{*}$ Correspondence:}

Roberto Weigert, Intracellular

Membrane Trafficking Unit, Oral and

Pharyngeal Cancer Branch, National

Institute of Dental and Craniofacial

Research, National Institutes of

Health, 30 Convent Dr. 303A,

Bethesda, MD 20892-4340, USA

e-mail: weigertr@mail.nih.gov

${ }^{\dagger}$ These authors have contributed equally to this work.
Non viral-mediated transfection of plasmid DNA provides a fast and reliable way to express various transgenes in selected cell populations in live animals. Here, we show an improvement of a previously published method that is based on injecting plasmid DNA into the ductal system of the salivary glands in live rats. Specifically, using complexes between plasmid DNA and polyethyleneimine (PEI) we show that the expression of the transgenes is directed selectively to the salivary acinar cells. PEI does not affect the ability of cells to undergo regulated exocytosis, which was one of the main drawbacks of the previous methods. Moreover PEI does not affect the proper localization and targeting of transfected proteins, as shown for the apical plasma membrane water channel aquaporin 5 (AQP5). Overall, this approach, coupled with the use of intravital microscopy, permits to conduct localization and functional studies under physiological conditions, in a rapid, reliable, and affordable fashion.

Keywords: intravital microscopy, in vivo transfection, polyethyleneimine, aquaporin 5, non-viral gene delivery, salivary glands, acinar cells

\section{INTRODUCTION}

Intravital microscopy (IVM) has enabled researchers to image several biological processes in live multicellular organisms and in real time (Pittet and Weissleder, 2011; Ritsma et al., 2012; Weigert et al., 2013). In the last decade, this approach has permitted to image subcellular organelles in live rodents with temporal and spatial resolution almost identical to that achieved in cell cultures (Dunn et al., 2002; Weigert et al., 2013; Weigert, 2014). This has been possible by the development of procedures aimed at minimizing the motion artifacts due to the heartbeat and respiration, which otherwise would make imaging at a submicron scale an impossible task. In particular, we have developed a rodent salivary gland model that has enabled (1) tracking individual structures for extended periods of time, and (2) extracting quantitative data in a rigorous fashion. This approach has been used to study various aspects of the dynamics of endocytosis and regulated exocytosis in the salivary epithelium of both rats and mice (Masedunskas and Weigert, 2008; Masedunskas et al., 2011a, 2012), and recently, it has revealed a novel modality of mitochondrial metabolism that cannot be observed in in vitro models (Porat-Shliom et al., 2014).

So far, pharmacological tools have been the main approach to study the molecular mechanisms regulating the dynamics of subcellular events in live animals (Sandoval et al., 2004; Masedunskas et al., 2011a). One of the current challenges is to generate molecular tools that can be rapidly expressed in vivo, and interfere with the process of interest. An elegant approach is to develop transgenic mice engineered to express fluorescently tagged molecules, which act as reporters for the pathway of interest, and, at the same time, lack or express the specific molecule under investigation. Although the application of gene editing techniques to mice (e.g., zinc finger or CRISPR is rapidly growing (Sung et al., 2012; Yang et al., 2013), these methods are still expensive and time consuming. Viable alternatives are in vivo transient transfection of transgenes and/or shRNAs, which can be accomplished by either viral or non-viral mediated delivery (Boulaiz et al., 2005; Kawakami et al., 2008; Wang et al., 2013). Viral-mediated gene transfer is the most widely used approach since it allows to transfect a high number of cells and to efficiently deliver and express the transgenes in non-dividing or slowly dividing cells (Boulaiz et al., 2005). This approach provides with the opportunity to perform biochemical analysis on the transfected tissues. In small animals, the main viral vectors currently used are: recombinant adenovirus serotype 5 (rAD5), adenoassociated virus serotype 2 (AAV2), and lenti viruses (Snyder and Francis, 2005; Adriaansen et al., 2010; Wilson et al., 2013). However, viral-mediated transfections have some limitations, which include adverse reactions due to the host immune response (Sun et al., 2003). Moreover, the generation of viruses is time consuming and subjected to safety restrictions. To overcome these issues, non viral-mediated techniques have been attempted in various organs since the early 90 's, and in particular, direct injections of plasmid DNA in rodent skeletal muscle have proven to be quite successful (Wolff et al., 1992). Following up on early studies in SGs (Goldfine et al., 1997), we have developed and characterized a transfection method that can be used with commercially available 
plasmids, and thus suitable for rapid screenings and mutation analysis. We have shown that plasmid DNA can be transfected in the SGs of live rats using three different modalities of administration, which ensure to direct the expression of the transgene to selected cell populations (Sramkova et al., 2009, 2012). Although the yield of non viral-based transfections is an order of magnitude lower than viral-based methods, this approach is robust and enables performing single cell imaging in vivo. Here we show a variation of one of these transfection methods that is based on the use of polyethyleneimine (PEI) a molecule previously used as a vehicle to compact and deliver siRNA in vivo (Kircheis et al., 2001; Nimesh, 2012). This approach permits the selective transfection of acinar cells in the SG epithelium in a rapid and reliable fashion without affecting the properties and the localization of the transgenes.

\section{RESULTS AND DISCUSSION \\ PEI FACILITATES THE TARGETING OF THE PLASMID DNA TO THE ACINAR CELLS OF THE SALIVARY GLANDS IN VIVO}

We have previously shown that plasmid DNA can be delivered to the submandibular SGs in live rats by retro-injection trough the salivary duct (or Wharton's duct). Plasmid DNA is loaded into the ductal system and the acinar canaliculi, thus gaining access to the salivary epithelium from the apical plasma membrane (APM) (Figure 1A). Specifically, we have shown that although plasmid DNA is uptaken by all the cells in the epithelium, only few of the proliferating cells of the intercalated ducts express the transgenes (Sramkova et al., 2012) (Figures 1B,F). On the other hand, plasmid DNA mixed with empty rAD5 particles elicited the expression of the transgenes primarily in large ducts (Sramkova et al., 2009). Finally, we have shown that plasmid DNA is targeted exclusively to the acinar cells during $\beta$ adrenergic-mediated stimulation of regulated exocytosis (Sramkova et al., 2009). Indeed, during secretion, plasmid DNA is internalized into the acinar cells by virtue of compensatory endocytosis that is elicited to maintain the homeostasis of the APM (Sramkova et al., 2009; Masedunskas et al., 2011b) (Figures 1B,F). However, one of the main drawbacks of this approach is the fact that $\beta$-adrenergic stimulation induces an extensive degranulation of the acinar cells (Masedunskas et al., 2011a) (Figure 1C, central panel). Since the biogenesis of the secretory granules is a slow process, their full complement is reconstituted in $24-36 \mathrm{~h}$, which is beyond the optimal expression of the transgene (16-24 from the administration of the plasmid). Therefore, this method cannot not be used to study processes such as regulated exocytosis, and trafficking from the TGN, where the granules are originated. To overcome this issue we sought to establish a method that could enhance the low level of endocytosis that occurs at the APM of the acinar cells without inducing exocytosis (Sramkova et al., 2012). Specifically, we tested PEI, which has been extensively used to deliver siRNAs both in vitro and in vivo. Although its precise mechanisms of action is not fully understood, PEI has been suggested to: (1) facilitate the packing of nucleotide strands, (2) increase the endocytosis of the complex PEI-siRNA, and (3) promote endosomal rupture, thus releasing the complex into the cytoplasm (Neu et al., 2005; Creusat et al., 2010). PEI was mixed with plasmid DNA encoding for the monomeric YFP (see

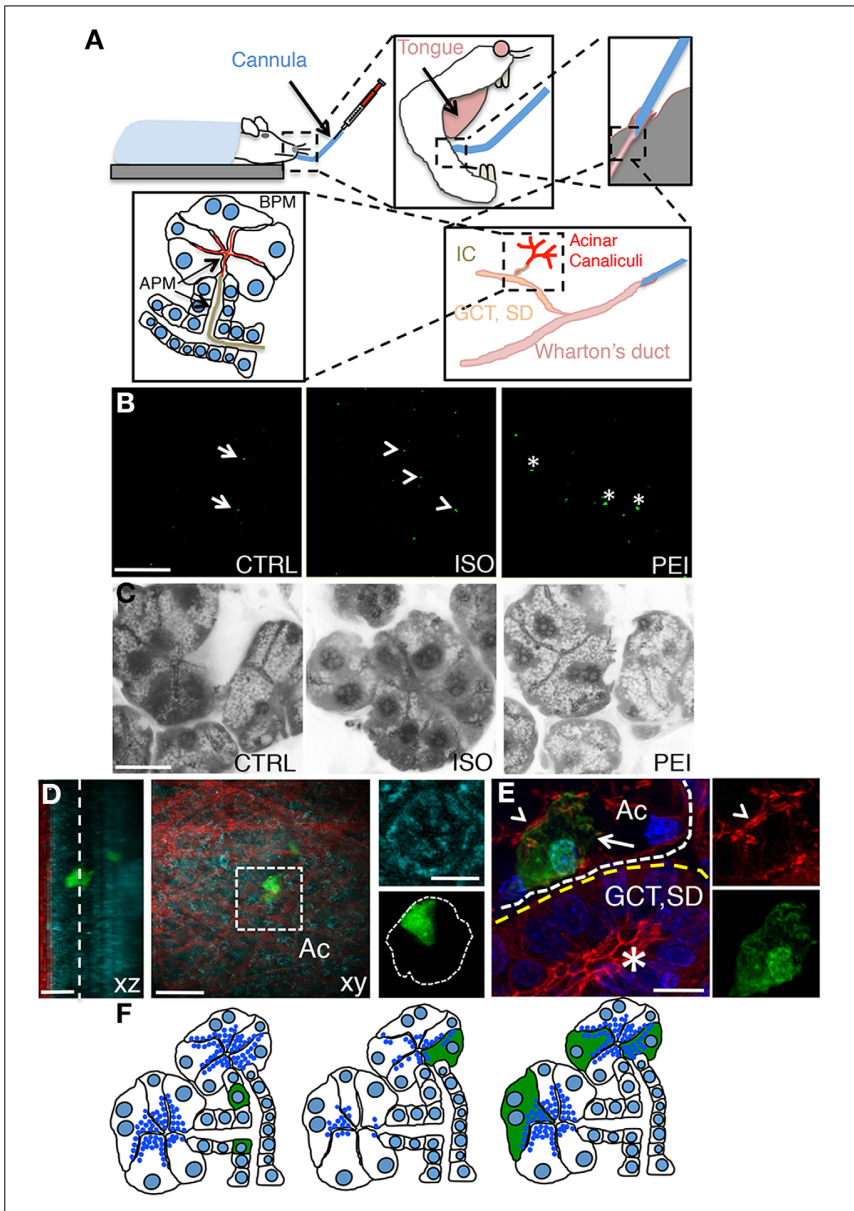

FIGURE 1 | Non viral-mediated expression of transgenes in rat salivary glands. (A) Diagram of the transfection procedures in anesthetized rats. A thin polyethylene cannula is inserted into the Wharton's duct, as described in the Methods sections. The plasmid DNA is gently injected and diffuses into the large ducts (granular convoluted tubules or GCT, and the striated ducts or SD), then into the intercalated ducts (IC), and finally into the acinar canaliculi. Plasmid DNA accesses the epithelium through the apical plasma membrane (APM, arrows). (B) In vivo transfection with plasmid DNA -12 $\mu \mathrm{g}$ of Plasmid DNA encoding for pVenus were injected alone (CTRL, left panel), after SC injection of isoproterenol (ISO, center panel), or mixed with PEI as described in the Methods (PEI, right panel). After $16 \mathrm{~h}$ the submandibular SGs were excised and immediately imaged by two-photon microscopy using a 10X objective (excitation $930 \mathrm{~nm}$ ) to reveal the cells expressing pVenus (arrows). Scale bar, $1 \mathrm{~mm}$. (C) Effect of PEI on regulated exocytosis. Mice expressing cytoplasmic GFP were treated as described in B and the SGs imaged by confocal microscopy (excitation $488 \mathrm{~nm}$ ) to estimate exocytosis of the secretory granules, as previously reported (Milberg et al., 2013). In the inset the apical plasma membrane (arrows) and the secretory granules (arrowheads) are highlighted. Scale bar, $20 \mu \mathrm{m}$. (D-E)

$\mathrm{PEl}$-mediated transfection in SGs drives expression into acinar cells. (D) Excised glands transfected with pVenus/PEI were imaged by two-photon microscopy using a 60x water immersion objective (D) or processed for immunofluorescence and imaged by confocal microscopy (E). (D) Two Z-stacks from the same area of the sample were acquired twice by using two different excitation wavelengths, respectively: $740 \mathrm{~nm}$ to reveal the epithelium of the gland (cyan) and $930 \mathrm{~nm}$ to reveal pVenus (green) and collagen fibers (red). The Z-stacks were combined and shown as xz (left) or xy (center) projection. The cell expressing pVenus is part of an acinus (inset, right). Scale bar, $20 \mu \mathrm{m}$. (E) The SGs were labeled for phallodin (red) and the nuclear staining Hoechst (blue). The cell expressing pVenus (green) is

(Continued) 


\section{FIGURE 1 | Continued}

part of an acinus (white broken line) as identified by the actin-labeled acinar canaliculi (arrowhead). Large ducts (yellow broken line), as identified by the characteristic actin pattern (asterisk) did not express any pVenus. Scale bar, $10 \mu \mathrm{m}$. (F) Diagram summarizing the pattern of expression of plasmid DNA in SGs. pVenus was expressed in cells of the IC under control conditions (left) and in acinar cells either upon stimulation with ISO (center) or administration with PEI (right).

Materials and Methods) and injected through the Wharton's duct of anesthetized rats. After $16 \mathrm{~h}$ the animals were anesthetized and the SGs were exposed and imaged by intravital two-photon microscopy (Figure 1B). Using a low magnification objective we sampled large areas of the tissue and observed that $\approx 10$ cells $/ \mathrm{mm}^{2}$ expressed the transgene, a level comparable to that observed in $\beta$ adrenergic- stimulated rats (Figure 2B). To determine whether PEI stimulated regulated exocytosis we used mice expressing cytoplasmic GFP that enable the visualization of the secretory granules (Masedunskas et al., 2011a; Milberg et al., 2013). Notably, SC injection of isoproterenol elicited robust exocytosis whereas PEI did not (Figure 1C and insets). The yield of transfection was maximal between 16-24 h from the administration (not shown). Moreover, we found that the transgene was expressed in acinar cells, as shown by performing either two-photon microscopy in freshly excised glands (Figure 1D) or immunocytochemistry and confocal microscopy (Figure 1E).

PEI facilitates the expression of molecules other than YFP, as shown by administering plasmid DNAs encoding for various molecules, such as cytoplasmic (e.g., actin, myosin Vc), nuclear (e.g., H2B), membrane-associated (e.g., Rab5), single and multiple spanning transmembrane proteins (e.g., TGN38, TfnR, VAMP8) (Figures 2A,B). The efficiency of transgene expression did not correlate with any characteristics of the expressed proteins or the size of the plasmid, as shown by expressing a transgene generated by fusing one, two or three copies of YFP (Figure 2C).

Our data show that PEI is an effective agent in facilitating the delivery and expression of transgenes into the acinar cells of rat salivary glands in vivo. Moreover, PEI does not cause any obvious cellular toxicity, as shown by the fact that regulated exocytosis and the dynamics of assembly of the actin cytoskeleton were not affected (Figure 2D). Finally, we did not observe any general signs of toxicity in the whole animal, at least within $24-48 \mathrm{~h}$ from the administration of PEI (not shown).

\section{PEI DOES NOT AFFECT PROTEIN TARGETING TO SPECIALIZED DOMAINS OF THE PLASMA MEMBRANE}

Next, we checked whether PEI affected the proper targeting of plasma membrane proteins to different domains of the acinar cells in the live animal. To this end, we investigated the expression and the localization of aquaporin 5 (AQP5), a water channel that regulates fluid secretion in mammalian SGs and is targeted to the APM via a sorting signal encoded in its C-terminus (Raina et al., 1995; Wellner et al., 2005; Delporte and Steinfeld, 2006; Horsefield et al., 2008).

Trafficking, localization, and targeting mechanism of AQP5 have been primarily investigated in cell cultures grown on solid substrates (Tada et al., 1999), in polarized MDCK cells (Nejsum and Nelson, 2007; Karabasil et al., 2009), and only to a small extent in native tissue in rodents (Gresz et al., 2004; Wellner et al., 2005; Matsuzaki et al., 2006). For these studies AQP5 has been tagged with fluorescent proteins (e.g., GFP or RFP) and the behavior of these constructs has been shown to be dependent on the experimental system and the position of the tag. We began by characterizing various constructs tagged either at the $\mathrm{C}$ - or the N-terminus (both human and rat AQP5) in cultured salivary glands-derived HSG cells (Royce et al., 1993). We used the monomeric form of the yellow fluorescent proteins (YFP) that has better quantum efficiency than the GFP when excited by 2-photon microscopy (Nagai et al., 2002). In HSG cells, we observed that AQP5-YFP was expressed but did not get exported out of the endoplasmic reticulum (Figure 3A, upper panel, arrows). On the other hand, YFP-AQP5 was properly targeted to the PM as previously reported (Wellner et al., 2005), and no intracellular vesicles were detected (Figure 3A, lower panel, arrowhead). Next, we generated stable HSG cells expressing YFP-AQP5 grown either as a confluent monolayer or in matrigel (Hoffman et al., 1996, 1998). In matrigel, HSG cells form acinar-like structures, which exhibit a partial polarization, as shown by phalloidin labeling (Figure 3B) and expression of amylase, a marker for acinar differentiation (Figure 3C) (Hoffman et al., 1996). YFP-AQP5 was properly targeted to the plasma membrane both in monolayers and in matrigel (Figure 3D). However, in acinar-like structures AQP5 did not exhibit any polarized localization, suggesting that other components may be required for the proper sorting, such as the $\mu 1-\mathrm{b}$ subunit of the AP1 adaptor complex (Figure 3D) (Ohno et al., 1999). Similar results were obtained expressing rat YFP-AQP5 (not shown).

Next, we injected the plasmid encoding for rat YFP-AQP5 into the salivary ducts of anesthetized rats by using PEI as vehicle. As expected, YFP-AQP5 was expressed in the SGs (Figure 4A, arrows), and primarily in acinar cells that were identified by endogenous fluorescence emissions (excitation $740 \mathrm{~nm}$ ), which highlight the cellular architecture of the salivary epithelium (Masedunskas and Weigert, 2008) (Figure 4A, lower panel). YFP-AQP5 was properly targeted to the APM as shown by two-photon microscopy (Figure 4B) and indirect immunofluorescence (Figure 4C, arrows). Interestingly, we observed that YFP-AQP5 was localized onto a series of intracellular vesicles localized in the sub-apical area of the acinar cells (Figures 4B,C, arrows) differently from what observed in cultured cells. These vesicles were not due to the fixation and the processing of the glands since they could be observed in the live animal (Figure 4B) and they were not an artifact of the ectopic expression of YFPAQP5, since endogenous AQP5 was also localized both at the APM and in vesicles (Figure 4C). Notably, a sub population of the YFP-AQP5 vesicles was found in close proximity of the TGN (Figures 4B,E). Although we could not determine whether these vesicles are endocytic or exocytic in nature, a time-lapse analysis revealed that they were stationary under basal conditions, possibly indicating that AQP5 is stored in an intracellular reservoir that may be mobilized during stimulation of water or protein secretion (Figure 4E), as shown for AQP2 in the kidney (Fushimi et al., 1997). 

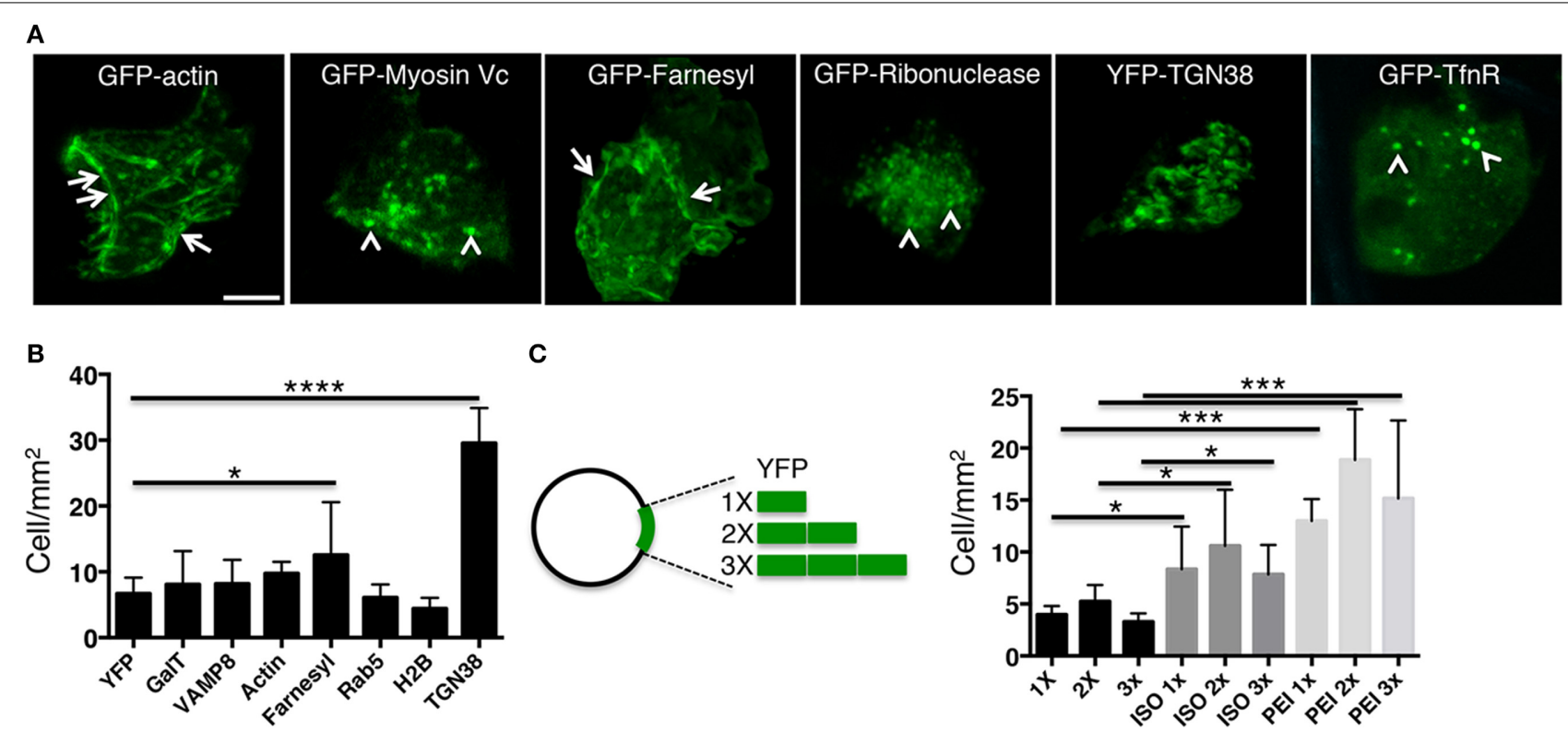

C

D
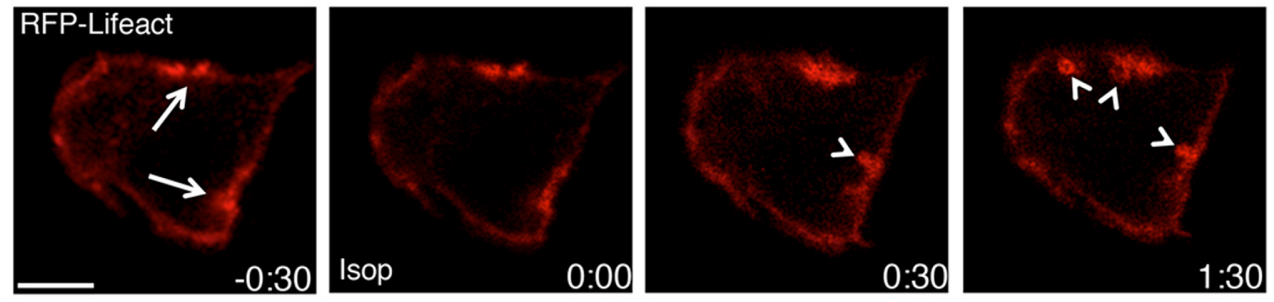

FIGURE 2 | PEI enables the expression of several transgenes in the acinar cells of the SGs. (A-C) Plasmid DNAs encoding either for selected fluorescently tagged proteins $(\mathbf{A}, \mathbf{B})$ or for multiple copies of pVenus (C) were injected into the SGs of anesthetized rats under different conditions. (A) High magnification images acquired by two-photon microscopy (excitation $930 \mathrm{~nm}$, 60x water-immersion objective) to reveal the intracellular localization of selected transfected transgenes. (B-C) The efficiency of expression of the transgene was determined as described in the Methods and expressed as cells $/ \mathrm{mm} 2$. Values represent average \pm S.D. $(N=3)$. Statistical significance was calculated by using a t-test $* p<0.1^{* * *} p<0.001 * * * * P<0.0001$. (B) Plasmids encoding for various cellular proteins and mixed with PEI are shown. (C) Plasmids containing $1(1 \mathrm{X}), 2(2 \mathrm{X})$ or $3(3 \mathrm{X})$ copies of pVenus fused together where injected into the salivary duct alone, after stimulation with ISO, or mixed with PEI. (D) Plasmid DNA encoding for the F-actin reporter RFP-lifeact was mixed with PEI and injected into the salivary duct of anesthetized rats. After $24 \mathrm{~h}$ the SGs were exposed and imaged by confocal microscopy (excitation $561 \mathrm{~nm}$ ). In acinar cells, RFP-life act was loclaized at the plasma membrane and in particular at the APM (arrows) SC injection of isoproterenol (time 0:00) elicited regulated exocytosis. As previously shown (Masedunskas et al., 2011a), secretory granules fused with the APM and recruited a F-actin coat (arrowheads). Scale bar, $10 \mu \mathrm{m}$.
In summary PEI-mediated in vivo transfection is a powerful tool to rapidly and reliably express transgenes in rat salivary glands in vivo. Although this method is suitable to perform single cell imaging and immunocytochemistry, its main limitation is the low yield of transfection (1-2\% of the cells of the parenchyma). Therefore, PEI is not suitable to carry out biochemical analysis or for gene therapy. Nonetheless, this approach provides the opportunity to perform localization and functional studies under physiological conditions using commercially available plasmids and in a relatively short time.

\section{MATERIALS AND METHODS \\ PLASMIDS AND CELL LINES}

Human AQP5 and rat AQP5 were obtained from Dr. Bruce Baum (NIDCR, NIH) and subcloned in the following vectors pEGFP-C1, pEGFP-N1, pEYFP-C1, pEYFP-N1 (Clontech). TGN38-mCherry and YFP-TGN38 were a generous gift from
Dr. Sarah Hamm-Alvarez (UCSC). GFP-actin, GFP-myosin Vc and GFP-TfnR were obtained from Dr. Julie Donaldson (NHLBI, NIH). GFP-Ribonuclease was a generous gift from Dr. David Yule (Rochester University). The 2X-EYFP vector was generated by inserting a copy of the YFP into a pEYFP-C and used to generate the $3 \mathrm{X}$-EYFP using the same procedure. HSG-cell lines stably expressing YFP-AQP5 were prepared by lentiviral expression, as previously reported (Amornphimoltham et al., 2013).

\section{CELL CULTURES}

HSG cells were cultured in Dulbecco's modified Eagle's medium/Ham's F-12 (1:1) (Invitrogen, CA) in the presence of 5\% fetal bovine serum, $100 \mathrm{U} / \mathrm{mL}$ penicillin, and $100 \mathrm{mg} / \mathrm{mL}$ streptomycin (Sigma-Aldrich, $\mathrm{MO}$ ) at $37^{\circ} \mathrm{C}$ in a $5 \% \mathrm{CO}_{2}$ humidified atmosphere.

Growth factor-reduced matrigel (Trevigen, MD) was diluted in complete medium at the appropriate concentration 

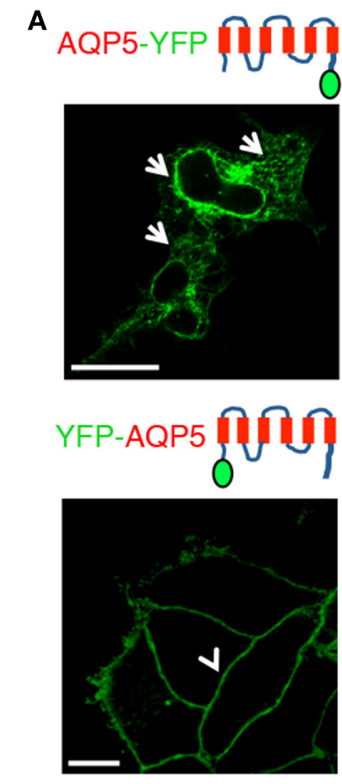

B
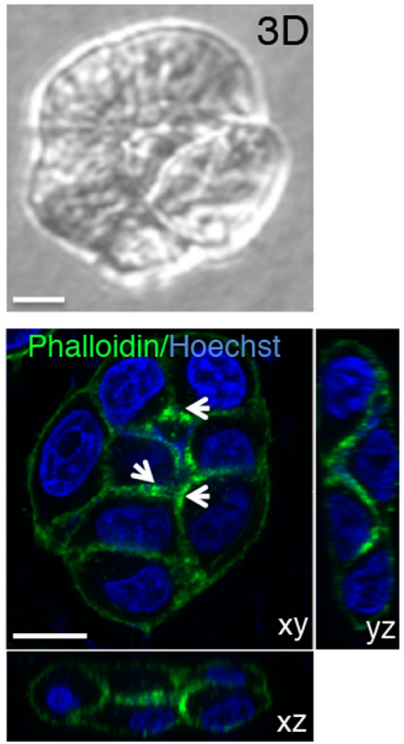

FIGURE 3 | Characterization of the fluorescently tagged AQP5. (A) Human AQP5 was tagged either at the (C) or the $\mathrm{N}$-terminus and transiently expressed in HSG cells grown on glass slides. AQP5-YFP is localized in the ER and the nuclear envelope (arrows), whereas YFP-AQP5 is localized at the APM (arrowheads). (B-D) HSG cells (B, C, and D lower panel) or HSG cells stably expressing YFP-AQP5 (D, upper panel) were grown for $96 \mathrm{~h}$ in matrigel to form acinar-like structures or on glass coverslips (C, upper panel) and imaged by
C

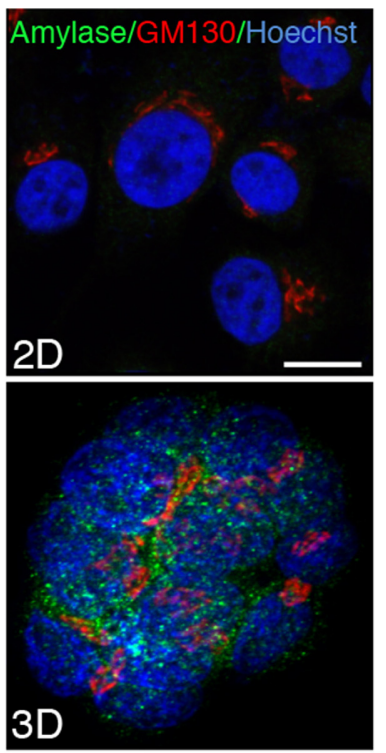

D
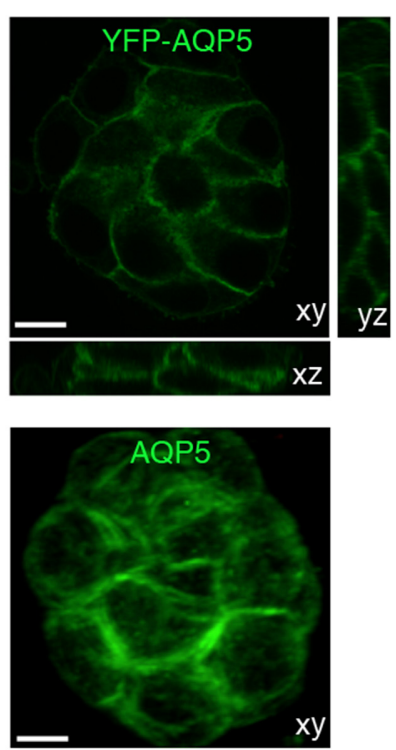

either phase contrast (B, upper panel) or confocal microscopy (B, C, lower panel and $\mathbf{C}$ ) in order to acquire Z-stacks. Cells were fixed and processed to reveal F-actin and the nuclei (B, lower panel), YFP-AQP5 (C, upper panel), endogenous AQP5 (C, lower panel), amylase (C) and the Golgi apparatus $(\mathrm{GM} 130, \mathbf{C})$. Maximal projections of the xy view and side views ( $\mathrm{xz}, \mathrm{yz}$ ) show that F-actin is partially polarized in the acinar-like cells (arrows) whereas both endogenous and ectopically expressed AQP5 did not. Scale bars, $5 \mu \mathrm{m}$.

(2.0-6.0 $\mathrm{mg} / \mathrm{mL})$, added to a 12-well tissue culture dish, and incubated for $1 \mathrm{~h}$ at $37^{\circ} \mathrm{C}$ before culturing of the cells. 50 cells $/ \mathrm{mm}^{2}$ were added onto the pre-solidified matrigel and grown for different times. Acinar cells were recovered from the matrigel by incubating in cell recovery solution (Trevigen, MD) for $30 \mathrm{~min}$ on ice. Acinar cells released from the matrigel were spread on glass coverslips and dehydrated at $37^{\circ} \mathrm{C}$ for $15 \mathrm{~min}$. Cells were then fixed in $2 \%$ formaldehyde/PBS, incubated first in blocking solution (10\% FBS, $0.02 \%$ sodium azide in PBS) for $15 \mathrm{~min}$, and later with various primary antibodies for $1 \mathrm{~h}$ at RT. Cells were then washed and incubated with the appropriate secondary antibodies for $1 \mathrm{~h}$ at RT. After 3 washes with blocking solution, nuclei were labeled by incubating the cells with Hoechst 33342 before mounting.

\section{ANIMAL PROCEDURES}

All the experiments were approved by the National Institute of Dental and Craniofacial Research (NIDCR, National Institute of Health, Bethesda, MD, USA) Animal Care and Use Committee. Sprague-Dawley male rats weighing 150-250 g were obtained from Harlan Laboratories Inc. (Frederick, MD) whereas the mice expressing cytoplasmic GFP were from Jackson laboratory (Masedunskas et al., 2011a). The animals were acclimated for 1 week before used for the procedures. Water and food were provided ad libitum. The animals were anesthetized by an IM injection of a mixture of Ketamine and Xylazine $(100 \mathrm{mg} / \mathrm{Kg}$ an $20 \mathrm{mg} / \mathrm{Kg}$ respectively) with additional injections as needed.

\section{PEI-PLASMID DNA COMPLEX}

Polyethyleneimine (PEI) was purchased from Polyplus Transfection (New York, NY) as in vivo-jet PEI. Efficient transfection is achieved by using $12-24 \mu \mathrm{g}$ of plasmid DNA/gland. Mix $50 \mu \mathrm{l}$ of $10 \%$ glucose with the plasmid DNA and adjust the volume to $100 \mu \mathrm{l}$ (solution 1). Mix $50 \mu \mathrm{l}$ of $10 \%$ glucose with $7.5 \mu \mathrm{l}$ of Jet PEI and adjust the volume to $100 \mu \mathrm{l}$ (solution 2). Mix solution 1 and solution 2 and incubate them for $30 \mathrm{~min}$ at room temperature. Aspirate the transfection mixture with a syringe (30-gage needle) and make sure that no air bubbles are released when injecting the fluid.

\section{IN VIVO TRANSFECTION}

Anesthetized rats were secured into a previously described stereotactic device (Masedunskas et al., 2013) with the mandibles wide open and the cheeks extended to the sides. The tongue was folded toward the back of the mouth to expose the ductal orifices without obstructing the airways. The stereotactic device was at about 45 degrees and positioned under a stereo-microscope (SZX7, Olympus America, Center Valley, PA) to visualize the area below the tongue and locate the two orifices of the Wharton's ducts. A 30-40 cm PE-5 cannula (Strategic Applications, Libertyville, IL) was gently pushed into the orifice and introduced into the duct using bent sharp tweezers. To seal the cannula a small drop of histoacryl tissue glue was applied to the orifice. The cannula was connected to the syringe containing the transfection mixture that was injected e gradually over a 5 min time period by applying gentle pressure on the plunger. The cannula was removed from the 


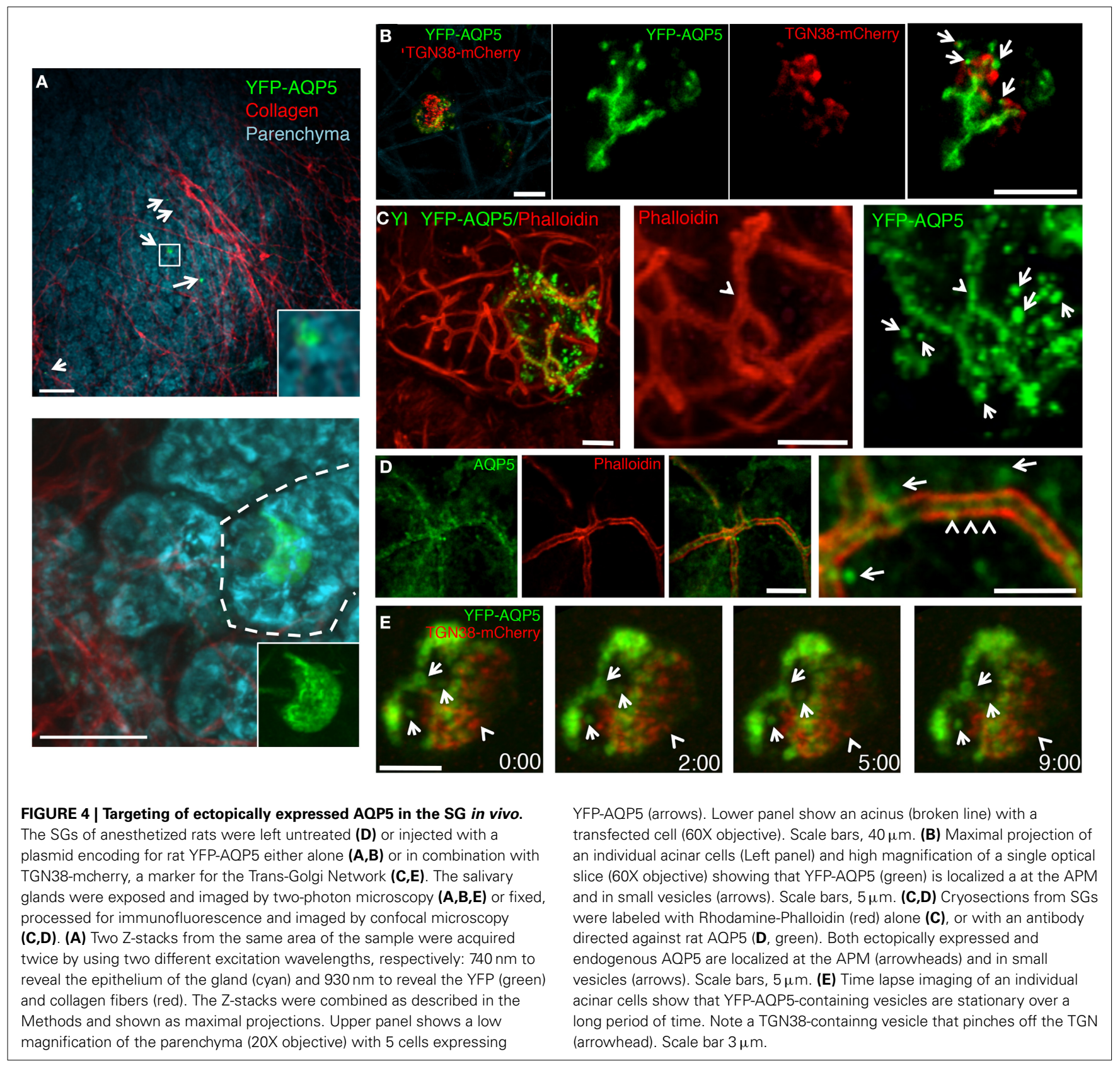

mouth and the animal was allowed to recover. A warm environment was provided to facilitate the recovery and the animal was monitored for at least $2 \mathrm{~h}$. The optimal yield of transfection was after 16-24 from the injection.

\section{TWO-PHOTON AND CONFOCAL MICROSCOPY}

Two-photon and confocal microscopy were performed on an IX81 inverted confocal microscope (Olympus, Melville, NY) modified to perform two-photon microscopy, as described previously (Masedunskas and Weigert, 2008). Intact and excised glands, and cultured cells, were imaged in the inverted setting by using the following objectives: UPLSAPO x10 NA 0.4, XLUMPFL20XW, 20X N.A. 0.95, and UPLSAPO 60X NA 1.2
(Olympus America Inc., Center Valley, PA). Intravital time-lapse imaging (Figure 4E) was performed as described in Masedunskas et al. (2011a) (acquisition speed 1 frame/sec).

\section{IMMUNOFLUORESCENCE}

The excised SGs were placed in optimum cutting temperature compound (Sakura Finetek USA Inc., Torrance, CA) and snap frozen in 2-methylbutane and liquid nitrogen. The glands were cut on silanated glass slides ( $10 \mu \mathrm{m}$ thickness) and cryosections were incubated with blocking solution for $45 \mathrm{~min}$ followed by incubation with the appropriate primary antibody or with TexasRed-Phalloidin (Invitrogen). Antibody against aquaporin 5 was purchased from Alomone labs. 


\section{ACKNOWLEDGMENTS}

This research was supported by the Intramural Research Program of the NIH, National Institute of Dental and Craniofacial Research. We thank Dr. Natalie Porat-Shliom for the critical reading of the manuscript.

\section{REFERENCES}

Adriaansen, J., Zheng, C., Perez, P., and Baum, B. J. (2010). Production and sorting of transgenic, modified human parathyroid hormone in vivo in rat salivary glands. Biochem. Biophys. Res. Commun. 391, 768-772. doi: 10.1016/j.bbrc.2009.11.135

Amornphimoltham, P., Rechache, K., Thompson, J., Masedunskas, A., Leelahavanichkul, K., Patel, V., et al. (2013). Rab25 regulates invasion and metastasis in head and neck cancer. Clin. Cancer Res. 19, 1375-1388. doi: 10.1158/1078-0432.CCR-12-2858

Boulaiz, H., Marchal, J. A., Prados, J., Melguizo, C., and Aranega, A. (2005). Nonviral and viral vectors for gene therapy. Cell. Mol. Biol. (Noisy-le-grand). 51, $3-22$.

Creusat, G., Rinaldi, A. S., Weiss, E., Elbaghdadi, R., Remy, J. S., Mulherkar, R., et al. (2010). Proton sponge trick for $\mathrm{pH}$-sensitive disassembly of polyethylenimine-based siRNA delivery systems. Bioconjug. Chem. 21, 994-1002. doi: 10.1021/bc100010k

Delporte, C., and Steinfeld, S. (2006). Distribution and roles of aquaporins in salivary glands. Biochim. Biophys. Acta 1758, 1061-1070. doi: 10.1016/j.bbamem.2006.01.022

Dunn, K. W., Sandoval, R. M., Kelly, K. J., Dagher, P. C., Tanner, G. A., Atkinson, S J., et al. (2002). Functional studies of the kidney of living animals using multicolor two-photon microscopy. Am. J. Physiol. Cell Physiol. 283, C905-C916. doi: 10.1152/ajpcell.00159.2002

Fushimi, K., Sasaki, S., and Marumo, F. (1997). Phosphorylation of serine 256 is required for cAMP-dependent regulatory exocytosis of the aquaporin2 water channel. J. Biol. Chem. 272, 14800-14804. doi: 10.1074/jbc.272.23. 14800

Goldfine, I. D., German, M. S., Tseng, H. C., Wang, J., Bolaffi, J. L., Chen, J. W., et al. (1997). The endocrine secretion of human insulin and growth hormone by exocrine glands of the gastrointestinal tract. Nat. Biotechnol. 15, 1378-1382. doi: $10.1038 /$ nbt1297-1378

Gresz, V., Kwon, T. H., Gong, H., Agre, P., Steward, M. C., King, L. S., et al. (2004) Immunolocalization of AQP-5 in rat parotid and submandibular salivary glands after stimulation or inhibition of secretion in vivo. Am. J. Physiol. Gastrointest. Liver Physiol. 287, G151-G161. doi: 10.1152/ajpgi.00480.2003

Hoffman, M. P., Kibbey, M. C., Letterio, J. J., and Kleinman, H. K. (1996). Role of laminin-1 and TGF-beta 3 in acinar differentiation of a human submandibular gland cell line (HSG). J. Cell Sci. 109(Pt 8), 2013-2021.

Hoffman, M. P., Nomizu, M., Roque, E., Lee, S., Jung, D. W., Yamada, Y., et al. (1998). Laminin-1 and laminin-2 G-domain synthetic peptides bind syndecan1 and are involved in acinar formation of a human submandibular gland cell line. J. Biol. Chem. 273, 28633-28641. doi: 10.1074/jbc.273.44.28633

Horsefield, R., Norden, K., Fellert, M., Backmark, A., Tornroth-Horsefield, S., Terwisscha van Scheltinga, A. C., et al. (2008). High-resolution x-ray structure of human aquaporin 5. Proc. Natl. Acad. Sci. U.S.A. 105, 13327-13332. doi: 10.1073/pnas.0801466105

Karabasil, M. R., Hasegawa, T., Azlina, A., Purwanti, N., Purevjav, J., Yao, C., et al. (2009). Trafficking of GFP-AQP5 chimeric proteins conferred with unphosphorylated amino acids at their PKA-target motif ((152)SRRTS) in MDCK-II cells. J. Med. Invest. 56, 55-63. doi: 10.2152/jmi.56.55

Kawakami, S., Higuchi, Y., and Hashida, M. (2008). Nonviral approaches for targeted delivery of plasmid DNA and oligonucleotide. J. Pharm. Sci. 97, 726-745. doi: $10.1002 /$ jps. 21024

Kircheis, R., Wightman, L., and Wagner, E. (2001). Design and gene delivery activity of modified polyethylenimines. Adv. Drug Deliv. Rev. 53, 341-358. doi: 10.1016/S0169-409X(01)00202-2

Masedunskas, A., Porat-Shliom, N., Rechache, K., Aye, M. P., and Weigert, R. (2012). Intravital microscopy reveals differences in the kinetics of endocytic pathways between cell cultures and live animals. Cells 1, 1121-1132. doi: $10.3390 /$ cells 1041121

Masedunskas, A., Sramkova, M., Parente, L., Sales, K. U., Amornphimoltham, P., Bugge, T. H., et al. (2011a). Role for the actomyosin complex in regulated exocytosis revealed by intravital microscopy. Proc. Natl. Acad. Sci. U.S.A. 108, 13552-13557. doi: 10.1073/pnas.1016778108

Masedunskas, A., Sramkova, M., Parente, L., and Weigert, R. (2013). Intravital microscopy to image membrane trafficking in live rats. Methods Mol. Biol. 931, 153-167. doi: 10.1007/978-1-62703-056-4_9

Masedunskas, A., Sramkova, M., and Weigert, R. (2011b). Homeostasis of the apical plasma membrane during regulated exocytosis in the salivary glands of live rodents. Bioarchitecture 1, 225-229. doi: 10.4161/bioa.18405

Masedunskas, A., and Weigert, R. (2008). Intravital two-photon microscopy for studying the uptake and trafficking of fluorescently conjugated molecules in live rodents. Traffic 9, 1801-1810. doi: 10.1111/j.1600-0854.2008.00798.x

Matsuzaki, T., Ablimit, A., Suzuki, T., Aoki, T., Hagiwara, H., and Takata, K. (2006). Changes of aquaporin 5-distribution during release and reaccumulation of secretory granules in isoproterenol-treated mouse parotid gland. J. Electron Microsc. (Tokyo) 55, 183-189. doi: 10.1093/jmicro/dfl023

Milberg, O., Porat-Shliom, N., Tora, M., Parente, L., Masedunskas, A., and Weigert, R. (2013). "Intravital microscopy and its application to study regulated exocytosis in the exocrine glands of live rodents," in Methods in the Study of Exocytosis, ed P. Thorn (Springer).

Nagai, T., Ibata, K., Park, E. S., Kubota, M., Mikoshiba, K., and Miyawaki, A. (2002). A variant of yellow fluorescent protein with fast and efficient maturation for cell-biological applications. Nat. Biotechnol. 20, 87-90. doi: 10.1038/nbt0 102-87

Nejsum, L. N., and Nelson, W. J. (2007). A molecular mechanism directly linking E-cadherin adhesion to initiation of epithelial cell surface polarity. J. Cell Biol. 178, 323-335. doi: 10.1083/jcb.200705094

Neu, M., Fischer, D., and Kissel, T. (2005). Recent advances in rational gene transfer vector design based on poly(ethylene imine) and its derivatives. J. Gene Med. 7, 992-1009. doi: 10.1002/jgm.773

Nimesh, S. (2012). Polyethylenimine as a promising vector for targeted siRNA delivery. Curr. Clin. Pharmacol. 7, 121-130. doi: 10.2174/15748841280 0228857

Ohno, H., Tomemori, T., Nakatsu, F., Okazaki, Y., Aguilar, R. C., Foelsch, H., et al. (1999). Mu1B, a novel adaptor medium chain expressed in polarized epithelial cells. FEBS Lett. 449, 215-220. doi: 10.1016/S0014-5793(99)00432-9

Pittet, M. J., and Weissleder, R. (2011). Intravital imaging. Cell 147, 983-991. doi: 10.1016/j.cell.2011.11.004

Porat-Shliom, N., Chen, Y., Tora, M., Shitara, A., Masedunskas, A., and Weigert, R. (2014). In vivo tissue-wide synchronization of mitochondrial metabolic oscillations. Cell Rep. doi: 10.1016/j.celrep.2014.09.022

Raina, S., Preston, G. M., Guggino, W. B., and Agre, P. (1995). Molecular cloning and characterization of an aquaporin cDNA from salivary, lacrimal, and respiratory tissues. J. Biol. Chem. 270, 1908-1912. doi: 10.1074/jbc.270. 4.1908

Ritsma, L., Ponsioen, B., and van Rheenen, J. (2012). Intravital imaging of cell signaling in mice. Intravital 1, 2-10. doi: 10.4161/intv.20802

Royce, L. S., Kibbey, M. C., Mertz, P., Kleinman, H. K., and Baum, B. J. (1993). Human neoplastic submandibular intercalated duct cells express an acinar phenotype when cultured on a basement membrane matrix. Differentiation 52, 247-255. doi: 10.1111/j.1432-0436.1993.tb00637.x

Sandoval, R. M., Kennedy, M. D., Low, P. S., and Molitoris, B. A. (2004). Uptake and trafficking of fluorescent conjugates of folic acid in intact kidney determined using intravital two-photon microscopy. Am. J. Physiol. Cell Physiol. 287, C517-C526. doi: 10.1152/ajpcell.00006.2004

Snyder, R. O., and Francis, J. (2005). Adeno-associated viral vectors for clinical gene transfer studies. Curr. Gene Ther. 5, 311-321. doi: 10.2174/1566523054065066

Sramkova, M., Masedunskas, A., Parente, L., Molinolo, A., and Weigert, R. (2009). Expression of plasmid DNA in the salivary gland epithelium: novel approaches to study dynamic cellular processes in live animals. Am. J. Physiol. Cell Physiol. 297, C1347-C1357. doi: 10.1152/ajpcell.00262.2009

Sramkova, M., Masedunskas, A., and Weigert, R. (2012). Plasmid DNA is internalized from the apical plasma membrane of the salivary gland epithelium in live animals. Histochem. Cell Biol. 138, 201-213. doi: 10.1007/s00418-012-0959-7

Sun, J. Y., Anand-Jawa, V., Chatterjee, S., and Wong, K. K. (2003). Immune responses to adeno-associated virus and its recombinant vectors. Gene Ther. 10, 964-976. doi: 10.1038/sj.gt.3302039

Sung, Y. H., Baek, I. J., Seong, J. K., Kim, J. S., and Lee, H. W. (2012). Mouse genetics: catalogue and scissors. BMB Rep. 45, 686-692. doi: 10.5483/BMBRep.2012. 45.12.242 
Tada, J., Sawa, T., Yamanaka, N., Shono, M., Akamatsu, T., Tsumura, K., et al. (1999). Involvement of vesicle-cytoskeleton interaction in AQP5 trafficking in AQP5-gene-transfected HSG cells. Biochem. Biophys. Res. Commun. 266, 443-447. doi: 10.1006/bbrc.1999.1828

Wang, W., Li, W., Ma, N., and Steinhoff, G. (2013). Non-viral gene delivery methods. Curr. Pharm. Biotechnol. 14, 46-60. doi: 10.2174/138920113804805278

Weigert, R. (2014). Imaging the dynamics of endocytosis in live mammalian tissues. Cold Spring Harb. Perspect. Biol. 6:a017012. doi: 10.1101/cshperspect.a017012

Weigert, R., Porat-Shliom, N., and Amornphimoltham, P. (2013). Imaging cell biology in live animals: ready for prime time. J. Cell Biol. 201, 969-979. doi: $10.1083 /$ jcb. 201212130

Wellner, R. B., Hong, S., Cotrim, A. P., Swaim, W. D., and Baum, B. J. (2005). Modifying the $\mathrm{NH} 2$ and $\mathrm{COOH}$ termini of aquaporin-5: effects on localization in polarized epithelial cells. Tissue Eng. 11, 1449-1458. doi: 10.1089/ten.2005.11.1449

Wilson, A. A., Kwok, L. W., Porter, E. L., Payne, J. G., McElroy, G. S., Ohle, S. J., et al. (2013). Lentiviral delivery of RNAi for in vivo lineage-specific modulation of gene expression in mouse lung macrophages. Mol. Ther. 21, 825-833. doi: 10.1038/mt.2013.19

Wolff, J. A., Dowty, M. E., Jiao, S., Repetto, G., Berg, R. K., Ludtke, J. J., et al. (1992). Expression of naked plasmids by cultured myotubes and entry of plasmids into T tubules and caveolae of mammalian skeletal muscle. J Cell Sci. 103(Pt 4), 1249-1259.
Yang, H., Wang, H., Shivalila, C. S., Cheng, A. W., Shi, L., and Jaenisch, R. (2013). One-step generation of mice carrying reporter and conditional alleles by CRISPR/Cas-mediated genome engineering. Cell 154, 1370-1379. doi: 10.1016/j.cell.2013.08.022

Conflict of Interest Statement: The authors declare that the research was conducted in the absence of any commercial or financial relationships that could be construed as a potential conflict of interest.

Received: 14 August 2014; accepted: 17 December 2014; published online: 09 January 2015.

Citation: Sramkova M, Parente L, Wigand T, Aye M-P, Shitara A and Weigert R (2015) Polyethylenimine-mediated expression of transgenes in the acinar cells of rats salivary glands in vivo. Front. Cell Dev. Biol. 2:74. doi: 10.3389/fcell.2014.00074

This article was submitted to Membrane Traffic, a section of the journal Frontiers in Cell and Developmental Biology.

Copyright (c) 2015 Sramkova, Parente, Wigand, Aye, Shitara and Weigert. This is an open-access article distributed under the terms of the Creative Commons Attribution License (CC BY). The use, distribution or reproduction in other forums is permitted, provided the original author(s) or licensor are credited and that the original publication in this journal is cited, in accordance with accepted academic practice. No use, distribution or reproduction is permitted which does not comply with these terms. 